
\title{
28 Resarch Soure \\ Integrative Analyses of Genes and miRNAs Associated With Age-Related Sarcopenia
}

\section{Sangyeob Lee}

Gyeongsang national university hospital

Jun-II Yoo ( $\nabla$ furim@hanmail.net)

Gyeongsang national university hospital

\section{Research}

Keywords: Integrative analyses, COX7A1, NDUFB5, miRNAs, Sarcopenia, Aging

Posted Date: August 2nd, 2021

DOI: https://doi.org/10.21203/rs.3.rs-732253/v1

License: (c) (i) This work is licensed under a Creative Commons Attribution 4.0 International License. Read Full License 


\section{Abstract}

Background: Sarcopenia is an age-related disease with skeletal muscle loss, weakness, and functional impairment. The potential causes of sarcopenia including the programmed cell death of muscles, inflammation, reactive oxygen species, protein turnover, and mitochondrial dysfunction have been studied. The purpose of this study was to find differentially expressed miRNAs (DE-miRNAs) in the muscle samples of older people (GSE23527). In addition, we performed to identify new miRNA-mRNA regulatory network for treating sarcopenia

Methods: Gene expression profiles were obtained from microarray datasets (GSE8479 and GSE1428) of the vastus lateralis muscles of young and older male subjects. Dataset GSE23527 was derived from the platform of GPL10358 (LC_MRA-1001_miRHuman_11.0_080411) and contained microRNA arrays of 12 young muscle samples and 12 older muscle samples. The DEGs between the older and young GSE8479 and GSE1428 samples were identified using the online analysis tool imaGEO (https://imageo.genyo.es). Pathway and process enrichment analysis with the ontology sources in the KEGG pathway, GO biological processes, Reactome gene sets, WikiPathways, and CORUM were analyzed by Metascape. A PPI (proteinprotein interaction) network of DEGs was constructed using the Search Tool for Retrieval of Interacting Genes (STRING) app in Cytoscape software (version 1.6.0). GEO2R (https://www.ncbi.nlm.nih.gov) was used to select differentially expressed miRNAs (DE-miRNAs) in the GSE 23527 dataset.

Results: In the GSE8479 and GSE1428 datasets, a total of 81 DEGs were discovered, including four upregulated genes and 77 downregulated genes. The top 12 clusters and their representative enriched terms were identified using Metascape. A total of 79 nodes and 186 edges were predicted in the PPI network. One upregulated DE-miRNA (hsa-miR-450a-5p) and six downregulated DE-miRNAs (hsa-miR127-3p, hsa-miR-24-2-5p, hsa-miR-378a-5p, hsa-miR-532-5p, hsa-miR-487b-5p, and has-miR-487b-3p) were selected in the miRBase database. The MiRWalk online database was utilized for exploring 8017 genes that were selected as genes regulated by DE-miRNAs and six of them overlapped with hub genes. COX7A1 and NDUFB5 showed significantly low expression in sarcopenia patients compared to the controls. COX7B and PDHA1 also displayed low expression in sarcopenia patients, but expression of COX7A1 and NDUFB5 was not significant. TIMM8A and CS showed similar expression rates in both samples.

Conclusions: The present bioinformatics analysis showed that two target genes (COX7A1 and NDUFB5) were potentially downregulated in sarcopenia patients. These two genes could be the cause of sarcopenia with aging. In addition, present study showed that several miRNAs (hsa-miR-378a-5p, hsamiR-532-5p, hsa-miR-127-3p, and hsa-miR-24-2-5p) were identified as regulating the target genes. These results suggest that controlling the identified miRNAs could be a prospective strategy for treating sarcopenia by regulating the mRNA-miRNA network.

\section{Introduction}


Sarcopenia is an age-related disease with skeletal muscle loss, weakness, and functional impairment. ${ }^{1}$ As skeletal muscle typically comprises the largest amount of body protein, a reduction in muscle can lead to muscle injury, decreasing the voluntary muscle capacity. ${ }^{2}$ The potential cause of sarcopenia including the programmed cell death of muscles, inflammation, reactive oxygen species (ROS), protein turnover, and mitochondrial dysfunction has been studied. ${ }^{3-5}$ Because society is aging, age-related sarcopenia will become even more obvious. Therefore, it is necessary to research the mechanism and ways to improve treatment of the disease.

Undoubtedly, energy-producing related factors might be important because sarcopenia is a muscle disorder of aged people. In addition, sarcopenia is not just a single protein, gene, or system dysfunction. Thus identifying genome-wide transcription expression patterns to confirm general gene function is an efficient method. To investigate the molecular mechanisms of muscle loss with age, researchers have utilized various methods like oligonucleotide-based microarrays, gene expression changes in skeletal muscle, and cDNA arrays. ${ }^{6-9}$ These studies showed that the genes related to DNA damage repair, energy metabolism, mitochondrial function, and oxidative stress were involved in the differential expression. The results provided meaningful insight into sarcopenia, but there were indisputable limitations due to the sample size.

MicroRNAs (miRNAs) are composed of ubiquitous and small non-coding RNA molecules. They contain approximately 22 nucleotides and function in RNA silencing or the regulation of gene expression. ${ }^{10}$ Each individual miRNA can modulate diverse mRNAs, indicating their great potential for the treatment and prognosis of diseases. ${ }^{11} \mathrm{~A}$ number of studies suggested that the aging process might be regulated by miRNAs. ${ }^{12(p 17), 13-16}$ Especially, since mitochondrial dysfunction and oxidative damage are very important in age-related diseases, many researchers have studied these topics. For example, Schmidt and Khatri suggested that nuclear respiratory factor-1 (Nrf2) was regulated by miR-340-5p and miR-206, which is a key factor in regulating redox environments. ${ }^{17,18}$ Several mitochondrial (mito)miRs (-34a, $-146 a$, and -181 a) were also related to aging and directly controlled mitochondrial function by regulating mitochondrial protein expression. The loss of mitochondrial function results in oxidative stress, inflammation, and age-related diseases. ${ }^{19,20}$ These factors associated with the mitochondrial system might be related to sarcopenia due to age-related gene regulation, but the relationship is still poorly understood.

The purpose of this study was to find differentially expressed miRNAs (DE-miRNAs) in the muscle samples of older people (GSE23527). In addition, we performed to identify new miRNA-mRNA regulatory network for treating sarcopenia.

\section{Methods}

\subsection{Microarray Data Source}


First, the gene expression profiles were downloaded from the GEO (http://www.ncbi.nlm.nih.gov/geo) databases. We obtained the microarray datasets (GSE8479 and GSE1428) of the vastus lateralis muscle of young and older male subjects. The microarray of the GS8479 and GSE1428 datasets was based on GPL2700 (Sentrix HumanRef-8 Expression BeadChip) and GPL96 ([HG-U133A] Affymetrix Human Genome U133A Array). GSE8479 contained 12 young muscle samples and 13 older muscle samples, and GSE1428 contained 10 young muscle samples and 12 older muscle samples. Additionally, the GSE23527 dataset was derived from the GPL10358 platform (LC_MRA-1001_miRHuman_11.0_080411) and contained the microRNA arrays of 12 young muscle samples and 12 older muscle samples.

\subsection{Data Processing and Pathway Enrichment Analysis of GSE8479 and GSE1428}

The DEGs between the older and young GSE8479 and GSE1428 samples were detected using the imaGEO (https://imageo.genyo.es) online analysis tool. An False Discovery Rate (FDR) P-value of $<0.05$ was considered to indicate a DEG. We used the Metascape online database (http://metascape.org) to analyze the DEG enrichment of several pathways ( $\min$ overlap $=3$, P-value cutoff $=0.01$, min enrichment $=1.5$ ). Pathway and process enrichment analysis with ontology sources including the KEGG pathway, GO biological processes, Reactome gene sets, WikiPathways, and CORUM were analyzed by Metascape. The GO biological process was used to identify unique properties of the genomic data and KEGG was utilized for defining relationships, molecular interactions, and reaction networks of the DEGs. Reactome and WikiPathways are free pathway databases selected by experts and the scientific community. The CORUM databases are a resource of manually annotated protein complexes.

\subsection{Protein-Protein Interaction Network Analysis and Hub Gene Identification}

The PPI network of the DEGs was constructed using the Search Tool for Retrieval of Interacting Genes (STRING) app in Cytoscape software (version 1.6.0). Then, the PPI network was evaluated using the MCODE app in Cytoscape (degree cutoff $=2$, max. depth $=100$, node score cutoff $=0.2$, and k-core $=2$ ). The 20 hub genes of the DEGs were identified by CytoHubba, a Cytoscape plugin, using maximum correlation criterion (MCC).

\subsection{Prediction of Potential miRNA and Target Genes}

GEO2R (https://www.ncbi.nlm.nih.gov) was used to select the DE-miRNAs in the GSE 23527 dataset. Adjusted P-values of $<0.05|\log F C|>0.8$ were set as the cutoff criteria with force normalization.

The genes regulated by DE-miRNAs in GSE23527 were selected using miRWalk and miRBase (http://www.mirbase.org/) databases using scores of $>0.95$ in the random forest-based approach by executing the TarPmiR algorithm for miRNA target site prediction. The intersection of the genes regulated by the DE-miRNAs and hub genes of the DEGs were considered the target genes. The Venn diagram web tool (http://bioinformatics.psb.ugent.be/webtools/Venn) was used to identify the target genes. 


\subsection{Statistics}

Bioinformatic tools were used for most of the statistical analyses above. When using the imaGEO web tool, meta-analysis was performed using the effect size of the random-effects model. In GEO2R, the Benjamini and Hochberg false discovery rate method was selected by default.

\section{Results}

\subsection{Identification and Enrichment of Differentially Expressed Genes in Young and Older Muscle Samples}

In the GSE8479 and GSE1428 datasets, a total of 81 DEGs were discovered, including four upregulated genes and 77 downregulated genes (Fig. 1A). The specific results of the gene analysis are presented in Supplementary Table 1.

Functional and pathway enrichment analyses of the DEGs were performed using Metascape online tools. The top 12 clusters and their representative enriched terms were identified (Fig. 1B). The Reactome gene sets showed that the citric acid (TCA) cycle and respiratory electron transport were the most significant pathways. In addition, GO biological processes and KEGG pathway analyses showed that mitochondrion organization and cardiac muscle contraction were also major pathways. The enriched GO terms are described in Table 1. A network plot of the enriched terms is also presented with the best $p$-values from each of the 20 clusters. Terms with a similarity of $>0.3$ are connected by edges (Figs. 1C and 1D). 
Table 1

Top 12 clusters with their representative enriched terms

\begin{tabular}{|c|c|c|c|c|c|c|}
\hline GO & Category & Description & Count & $\%$ & $\log 10(P)$ & $\log 10(q)$ \\
\hline $\begin{array}{l}\text { R-HSA- } \\
1428517\end{array}$ & $\begin{array}{l}\text { Reactome } \\
\text { Gene Sets }\end{array}$ & $\begin{array}{l}\text { The citric acid (TCA) } \\
\text { cycle and respiratory } \\
\text { electron transport }\end{array}$ & 17 & 20.99 & -20.77 & -16.41 \\
\hline GO:0007005 & $\begin{array}{l}\text { GO Biological } \\
\text { Processes }\end{array}$ & $\begin{array}{l}\text { mitochondrion } \\
\text { organization }\end{array}$ & 16 & 19.75 & -11.45 & -8.43 \\
\hline hsa04260 & $\begin{array}{l}\text { KEGG } \\
\text { Pathway }\end{array}$ & $\begin{array}{l}\text { Cardiac muscle } \\
\text { contraction }\end{array}$ & 7 & 8.64 & -8.55 & -5.64 \\
\hline $\begin{array}{l}\text { R-HSA- } \\
1268020\end{array}$ & $\begin{array}{l}\text { Reactome } \\
\text { Gene Sets }\end{array}$ & $\begin{array}{l}\text { Mitochondrial protein } \\
\text { import }\end{array}$ & 6 & 7.41 & -7.51 & -4.68 \\
\hline hsa01200 & $\begin{array}{l}\text { KEGG } \\
\text { Pathway }\end{array}$ & Carbon metabolism & 6 & 7.41 & -6.01 & -3.26 \\
\hline G0:0032787 & $\begin{array}{l}\text { GO Biological } \\
\text { Processes }\end{array}$ & $\begin{array}{l}\text { monocarboxylic acid } \\
\text { metabolic process }\end{array}$ & 10 & 12.35 & -4.79 & -2.16 \\
\hline CORUM:320 & CORUM & $\begin{array}{l}\text { 55S ribosome, } \\
\text { mitochondrial }\end{array}$ & 4 & 4.94 & -4.14 & -1.6 \\
\hline hsa00020 & $\begin{array}{l}\text { KEGG } \\
\text { Pathway }\end{array}$ & $\begin{array}{l}\text { Citrate cycle (TCA } \\
\text { cycle) }\end{array}$ & 3 & 3.7 & -4.06 & -1.53 \\
\hline WP4255 & WikiPathways & $\begin{array}{l}\text { Non-small cell lung } \\
\text { cancer }\end{array}$ & 3 & 3.7 & -2.93 & -0.62 \\
\hline G0:0030901 & $\begin{array}{l}\text { GO Biological } \\
\text { Processes }\end{array}$ & midbrain development & 3 & 3.7 & -2.7 & -0.45 \\
\hline G0:0051341 & $\begin{array}{l}\text { GO Biological } \\
\text { Processes }\end{array}$ & $\begin{array}{l}\text { regulation of } \\
\text { oxidoreductase activity }\end{array}$ & 3 & 3.7 & -2.4 & -0.18 \\
\hline G0:0106106 & $\begin{array}{l}\text { GO Biological } \\
\text { Processes }\end{array}$ & $\begin{array}{l}\text { cold-induced } \\
\text { thermogenesis }\end{array}$ & 3 & 3.7 & -2.08 & 0 \\
\hline
\end{tabular}

\subsection{PPI Network and Module Analysis}

Using the STRING app in Cytoscape, the potential correlation between these DEGs was examined (maximum additional interactors $=0$, confidence score cutoff $=0.4$ ) (Fig. 2A). A total of 79 nodes and 186 edges were predicted in the PPI network. Then, the MCODE app in Cytoscape showed the significant modules in the PPI network (degree cutoff $=2$, max. depth $=100$, node score cutoff $=0.2$, and k-core $=2$ ) (Figs. 2B, 2C, and 2D). CytoHubba plugin in Cytoscape is used for clustering the hub genes (Fig. 2E). The top 20 hub genes are included in Table 2 with Maximum correlation criterion (MMC) scores. 
Table 2

Top 20 in network String Network ranked by MCC method.

\begin{tabular}{|c|c|c|c|}
\hline Rank & Name & shared name & Score \\
\hline 1 & 9606.ENSP00000317780 & $\operatorname{cox} 5 \mathrm{~A}$ & 39900000 \\
\hline 2 & 9606.ENSP00000306397 & UQCRFS1 & 39900000 \\
\hline 3 & 9606.ENSP00000367939 & UQCRQ & 39900000 \\
\hline 4 & 9606.ENSP00000457513 & $\operatorname{cox} 411$ & 39900000 \\
\hline 5 & 9606.ENSP00000359098 & COX7A2 & 39900000 \\
\hline 6 & 9606.ENSP00000419087 & NDUFB2 & 39900000 \\
\hline 7 & 9606.ENSP00000417656 & cox7B & 39900000 \\
\hline 8 & 9606.ENSP00000377033 & ATP5G1 & 39900000 \\
\hline 9 & 9606.ENSP00000284727 & ATP5G3 & 39900000 \\
\hline 10 & 9606.ENSP00000259037 & NDUFB5 & 39900000 \\
\hline 11 & 9606.ENSP00000389649 & ATP5J & 39900000 \\
\hline 12 & 9606.ENSP00000237889 & NDUFB3 & 39900000 \\
\hline 13 & 9606.ENSP00000292907 & COX7A1 & 5040 \\
\hline 14 & 9606.ENSP00000377446 & SUCLG1 & 1683 \\
\hline 15 & 9606.ENSP00000342056 & CS & 889 \\
\hline 16 & 9606.ENSP00000368528 & APOO & 850 \\
\hline 17 & 9606.ENSP00000401770 & C14orf2 & 744 \\
\hline 18 & 9606.ENSP00000369134 & PDHA1 & 173 \\
\hline 19 & 9606.ENSP00000361993 & TIMM8A & 49 \\
\hline 20 & 9606.ENSP00000387262 & IMMT & 44 \\
\hline
\end{tabular}

\subsection{Prediction of Potential miRNAs and Target Genes}

The microarray GSE23527expression dataset was analyzed using GEO2R. A total of seven DE-miRNAs were obtained (Fig. 3A volcano plot). Based on data from GEO2R, one upregulated DE-miRNA (hsa-miR450a-5p) and six downregulated DE-miRNAs (hsa-miR-127-3p, hsa-miR-24-2-5p, hsa-miR-378a-5p, hsamiR-532-5p, hsa-miR-487b-5p, and has-miR-487b-3p) were selected from the miRBase database for further analysis (Table 3). Full DE-miRNA data are included in Supplementary Table 2. 
Table 3

Selection of DE-miRNAs from miRBase.

\begin{tabular}{|llll|}
\hline DE-miRNAs & Regulation state & Selected DE-miRNAs from miRBase & Regulation state \\
\hline Ctr01-3M10 & Up & $x$ & $x$ \\
\hline hsa-miR-127-3p & Down & hsa-miR-127-3p & Down \\
\hline hsa-miR-24-2* & Down & hsa-miR-24-2-5p & Down \\
\hline hsa-miR-378* & Down & hsa-miR-378a-5p & Down \\
\hline hsa-miR-450a & Up & hsa-miR-450a-5p & Up \\
\hline hsa-miR-487b & Down & hsa-miR-487b-5p & Down \\
& & has-miR-487b-3p & Down \\
\hline hsa-miR-532-5p & Down & hsa-miR-532-5p & Down \\
\hline
\end{tabular}

The MiRWalk online database was utilized for exploring 8017 genes that were selected as genes regulated by DE-miRNAs (Supplementary Table 3 ) and six of them overlapped with the hub genes (Fig. 3B). These target genes all showed low expression in older people (Fig. 1A). These target genes were entered into the miRNA-target gene regulatory network in Cytoscape (Fig. 3C).

\section{Discussion}

Sarcopenia refers to gradual and progressive functional limitations and deterioration in muscle strength and endurance. ${ }^{21,22}$ Aging is a major factor in this disease by affecting physical activity capability and the molecular basis for the loss of muscle mass. ${ }^{23,24}$ To understand the underlying biology of sarcopenia and discover an effective intervention that can improve muscle function, it is necessary to monitor gene expression changes in a genome-wide study. Bioinformatics analyses including extended previous data are based on screening genetic alterations of gene expression. ${ }^{25}$

By identifying the key expression genes and miRNAs of disease including mRNA-miRNA interactions, many helpful disease control strategies can be acquired. To identify more optimal core genes of muscle weakness involved in aging, this study used two profile datasets (GSE8479 and GSE1428) based on bioinformatics methods. Twenty-five aged muscle specimens and twenty-two young muscle specimens were used in the research. Via imaGEO online analysis, a total of 81 commonly changed DEGs including four upregulated and 77 downregulated DEGs were revealed. After that, the Metascape online tool was used for functional and pathway enrichment analysis. DEGs were enriched in the category of 1) Reactome gene sets including the TCA cycle and respiratory electron transport and mitochondrial protein import; 2) GO biological processes including mitochondrion organization, monocarboxylic acid metabolic processes, midbrain development, and the regulation of oxidoreductase activity and cold-induced thermogenesis; 3) the KEGG pathway including cardiac muscle contraction, carbon metabolism, and the citrate cycle; and 4) CORUM including the mitochondrial 55S ribosome. Particularly, 23 DEGs including 
the citric acid (TCA) cycle, respiratory electron transport, and mitochondrial protein import were identified in the Reactome gene sets. In GO biological process and KEGG pathway analyses, mitochondrion organization and cardiac muscle contraction were most associated in each category. Next, a PPI network complex of the DEGs was constructed, which was composed of 79 nodes and 186 edges via Cytoscape using the STRING database. Three vital clusters were obtained by MCODE analysis including 1) cluster one of 12 nodes (COX7B, COX4I1, COX5A, COX7A2, ATP5J, ATP5G1, ATP5G3, NDUFB2, NDUFB3, NDUFB5, UQCRQ, and UQCRFS1) and 66 edges; 2 ) cluster two of four nodes (CS, APOO, SUCLG1, and PDHA1) and five edges; and 3) cluster three of three nodes (MRPL2, MRPL12, and MRPL34) and three edges. In CytoHubba, a Cytoscape plugin, 20 hub genes (COX5A, UQCRFS1, UQCRQ, COX4I1, COX7A2, NDUFB2, COX7B, ATP5G1, ATP5G3, NDUFB5, ATP5J, NDUFB3, COX7A1, SUCLG1, CS, AP00, C14orf2, PDHA1, and TIMM8A) were clarified. Seven DE-MiRNAs (hsa-miR-450a-5p, hsa-miR-127-3p, hsa-miR-24-25p, hsa-miR-378a-5p, hsa-miR-532-5p, hsa-miR-487b-5p, and has-miR-487b-3p) based on one profile dataset (GSE23527) were identified and the Venn diagram web tool was used for the target genes. Finally, we found six target genes (COX7A1, NDUFB5, COX7B, PDHA1, TIMM8A, and CS) and miRNAs that could be considered novel effective targets to treat patients with age-related sarcopenia.

The present study showed that most genes were related to mitochondrial function and energy-producing capabilities. Skeletal muscle mitochondrial capacity is an important factor and is well-studied. ${ }^{26,27}$ Human aging generally results in lower mitochondrial function. ${ }^{28-30}$ There are close links between muscle mass and mitochondrial energetics including reduced ATP ${ }^{31}$ and increased ROS generation. ${ }^{32,33}$ ROS produced in aged muscle results in proteolytic degradation (proteasome system) and energetic stress, leading to reduced muscle mass. ${ }^{34,35}$ If muscle mitochondria fail to provide sufficient ATP, the cells choose either growth or somatic maintenance, and this results in the disruption of proteostasis equilibrium. ${ }^{36}$

Because miRNAs are very competitive therapeutic molecules, our findings of miRNAs regulating mitochondrial function-related genes could be used in strategies for recovering the muscle atrophy of patients with age-related sarcopenia. ${ }^{15} \mathrm{~A}$ recent clinical trial indicated that specific inhibitors of miRNAs called anti-miR compounds represented potential drugs for incurable diseases ${ }^{37}$, and our study showed a new approach to treating sarcopenia, which remains a challenging muscle disorder.

\section{Conclusion}

The present study found that several miRNAs (hsa-miR-378a-5p, hsa-miR-532-5p, hsa-miR-127-3p, hsamiR-24-2-5p, has-miR-487b-5p, and has-miR-450a-5p) regulating target genes (COX7A1, NDUFB5, COX7B, PDHA1, TIMM8A, and CS) shows significant expression change in aged people. The bioinformatics analysis showed that the controlling miRNAs regulating mitochondrial function-related genes could be used in strategies for treating sarcopenia by regulating the mRNA-miRNA network. It is supposed that this mRNA-miRNA network could be the cause of sarcopenia with aging. These results suggest that the 
controlling miRNAs found could be used in strategies for treating sarcopenia by regulating the mRNAmiRNA network.

\section{Declarations}

\section{Ethics approval and consent to participate}

The study protocol was approved by the Institutional Review Board (IRB) of Gyeongsang National University Hspital (IRB number: 2019-02-013).

\section{Availability of data and materials}

The datasets generated and/or analyzed during the current study are available in the [GEO data, (GSE8479 and GSE1428] repository, [http://www.ncbi.nlm.nih.gov/geo].

\section{Competing interests}

None declared by all authors.

\section{Funding}

This work was funded by the by the National Research Foundation of Korea (NRF) grant funded by the Korea government (MEST) (No. NRF-2019R1F1A1059208). The National Research Foundation of Korea had no role in design or analysis of the project, or the writing of this article.

\section{Author's contributions}

Concept - YJI, LSY; Design - YJI, LSY; Supervision - YJI, LSY; Materials - YJI, LSY; Data Collection and/or Processing - YJI, LSY; Analysis and/or Interpretation - YJI, LSY; Literature Search - YJI, LSY; Writing Manuscript - YJI, LSY; Critical Review - YJI, LSY

\section{Acknowledgments}

Not Applicable

\section{References}

1. Doherty TJ. Invited review: Aging and sarcopenia. J Appl Physiol (1985). 2003;95(4):1717-1727. doi:10.1152/japplphysiol.00347.2003

2. Fried LP, Hadley EC, Walston JD, et al. From bedside to bench: research agenda for frailty. Sci Aging Knowledge Environ. 2005;2005(31):pe24. doi:10.1126/sageke.2005.31.pe24

3. Kujoth GC, Hiona A, Pugh TD, et al. Mitochondrial DNA mutations, oxidative stress, and apoptosis in mammalian aging. Science. 2005;309(5733):481-484. doi:10.1126/science.1112125 
4. Yasuda N, Glover El, Phillips SM, Isfort RJ, Tarnopolsky MA. Sex-based differences in skeletal muscle function and morphology with short-term limb immobilization. J Appl Physiol (1985). 2005;99(3):10851092. doi:10.1152/japplphysiol.00247.2005

5. Emelyanova L, Preston C, Gupta A, et al. Effect of Aging on Mitochondrial Energetics in the Human Atria. J Gerontol A Biol Sci Med Sci. 2018;73(5):608-616. doi:10.1093/gerona/glx160

6. Jozsi AC, Dupont-Versteegden EE, Taylor-Jones JM, et al. Aged human muscle demonstrates an altered gene expression profile consistent with an impaired response to exercise. Mech Ageing Dev. 2000;120(13):45-56. doi:10.1016/s0047-6374(00)00178-0

7. Larsson L, Grimby G, Karlsson J. Muscle strength and speed of movement in relation to age and muscle morphology. J Appl Physiol Respir Environ Exerc Physiol. 1979;46(3):451-456. doi:10.1152/jappl.1979.46.3.451

8. Welle S, Bhatt K, Thornton CA. High-abundance mRNAs in human muscle: comparison between young and old. J Appl Physiol (1985). 2000;89(1):297-304. doi:10.1152/jappl.2000.89.1.297

9. Welle S, Brooks Al, Delehanty JM, Needler N, Thornton CA. Gene expression profile of aging in human muscle. Physiological Genomics. 2003;14(2):149-159. doi:10.1152/physiolgenomics.00049.2003

10. Bartel DP. Metazoan MicroRNAs. Cell. 2018;173(1):20-51. doi:10.1016/j.cell.2018.03.006

11. Bartel DP. MicroRNAs: genomics, biogenesis, mechanism, and function. Cell. 2004;116(2):281-297. doi:10.1016/s0092-8674(04)00045-5

12. Hackl M, Brunner S, Fortschegger $\mathrm{K}$, et al. miR-17, miR-19b, miR-20a, and miR-106a are downregulated in human aging. Aging Cell. 2010;9(2):291-296. doi:10.1111/j.1474-9726.2010.00549.x

13. Ibáñez-Ventoso C, Yang M, Guo S, Robins H, Padgett RW, Driscoll M. Modulated microRNA expression during adult lifespan in Caenorhabditis elegans. Aging Cell. 2006;5(3):235-246. doi:10.1111/j.14749726.2006.00210.x

14. Liang R, Bates DJ, Wang E. Epigenetic Control of MicroRNA Expression and Aging. Curr Genomics. 2009;10(3):184-193. doi:10.2174/138920209788185225

15. van Rooij E, Purcell AL, Levin AA. Developing microRNA therapeutics. Circ Res. 2012;110(3):496-507. doi:10.1161/CIRCRESAHA.111.247916

16. Nie M, Deng Z-L, Liu J, Wang D-Z. Noncoding RNAs, Emerging Regulators of Skeletal Muscle Development and Diseases. Biomed Res Int. 2015;2015:676575. doi:10.1155/2015/676575

17. Schmidt M, Schüler SC, Hüttner SS, von Eyss B, von Maltzahn J. Adult stem cells at work: regenerating skeletal muscle. Cell Mol Life Sci. 2019;76(13):2559-2570. doi:10.1007/s00018-019-03093-6 
18. Khatri B, Seo D, Shouse S, et al. MicroRNA profiling associated with muscle growth in modern broilers compared to an unselected chicken breed. BMC Genomics. 2018;19(1):683. doi:10.1186/s12864-0185061-7

19. Rippo MR, Olivieri F, Monsurrò V, Prattichizzo F, Albertini MC, Procopio AD. MitomiRs in human inflamm-aging: a hypothesis involving miR-181a, miR-34a and miR-146a. Exp Gerontol. 2014;56:154-163. doi:10.1016/j.exger.2014.03.002

20. Giuliani A, Cirilli I, Prattichizzo F, et al. The mitomiR/Bcl-2 axis affects mitochondrial function and autophagic vacuole formation in senescent endothelial cells. Aging (Albany NY). 2018;10(10):2855-2873. doi:10.18632/aging.101591

21. Greenlund LJS, Nair KS. Sarcopenia--consequences, mechanisms, and potential therapies. Mech Ageing Dev. 2003;124(3):287-299. doi:10.1016/s0047-6374(02)00196-3

22. Mühlberg W, Sieber C. Sarcopenia and frailty in geriatricpatients: Implications for training andprevention. Z Gerontol Geriat. 2004;37(1):2-8. doi:10.1007/s00391-004-0203-8

23. Starling RD, Ades PA, Poehlman ET. Physical activity, protein intake, and appendicular skeletal muscle mass in older men. Am J Clin Nutr. 1999;70(1):91-96. doi:10.1093/ajcn/70.1.91

24. Goyns MH, Charlton MA, Dunford JE, et al. Differential display analysis of gene expression indicates that age-related changes are restricted to a small cohort of genes. Mech Ageing Dev. 1998;101(1-2):73-90. doi:10.1016/s0047-6374(97)00166-8

25. Mullighan CG, Goorha S, Radtke I, et al. Genome-wide analysis of genetic alterations in acute lymphoblastic leukaemia. Nature. 2007;446(7137):758-764. doi:10.1038/nature05690

26. Coen PM, Jubrias SA, Distefano G, et al. Skeletal muscle mitochondrial energetics are associated with maximal aerobic capacity and walking speed in older adults. J Gerontol A Biol Sci Med Sci. 2013;68(4):447-455. doi:10.1093/gerona/gls196

27. Gonzalez-Freire M, Scalzo P, D’Agostino J, et al. Skeletal muscle ex vivo mitochondrial respiration parallels decline in vivo oxidative capacity, cardiorespiratory fitness, and muscle strength: The Baltimore Longitudinal Study of Aging. Aging Cell. 2018;17(2). doi:10.1111/acel.12725

28. Tonkonogi M, Fernström M, Walsh B, et al. Reduced oxidative power but unchanged antioxidative capacity in skeletal muscle from aged humans. Pflugers Arch. 2003;446(2):261-269. doi:10.1007/s00424-003-1044-9

29. Lanza IR, Short DK, Short KR, et al. Endurance Exercise as a Countermeasure for Aging. Diabetes. 2008;57(11):2933-2942. doi:10.2337/db08-0349 
30. Porter C, Hurren NM, Cotter MV, et al. Mitochondrial respiratory capacity and coupling control decline with age in human skeletal muscle. American Journal of Physiology-Endocrinology and Metabolism. 2015;309(3):E224-E232. doi:10.1152/ajpendo.00125.2015

31. Romanello V, Guadagnin E, Gomes L, et al. Mitochondrial fission and remodelling contributes to muscle atrophy. EMBO J. 2010;29(10):1774-1785. doi:10.1038/emboj.2010.60

32. Kavazis AN, Talbert EE, Smuder AJ, Hudson MB, Nelson WB, Powers SK. Mechanical ventilation induces diaphragmatic mitochondrial dysfunction and increased oxidant production. Free Radic Biol Med. 2009;46(6):842-850. doi:10.1016/j.freeradbiomed.2009.01.002

33. Min K, Smuder AJ, Kwon O-S, Kavazis AN, Szeto HH, Powers SK. Mitochondrial-targeted antioxidants protect skeletal muscle against immobilization-induced muscle atrophy. J Appl Physiol (1985).

2011;111(5):1459-1466. doi:10.1152/japplphysiol.00591.2011

34. Hydrogen peroxide stimulates ubiquitin-conjugating activity and expression of genes for specific E2 and E3 proteins in skeletal muscle myotubes | American Journal of Physiology-Cell Physiology. Accessed May 17, 2021. https://journals.physiology.org/doi/full/10.1152/ajpcell.00129.2003

35. Jang YC, Lustgarten MS, Liu Y, et al. Increased superoxide in vivo accelerates age-associated muscle atrophy through mitochondrial dysfunction and neuromuscular junction degeneration. FASEB J. 2010;24(5):1376-1390. doi:10.1096/fj.09-146308

36. Hou C. The energy trade-off between growth and longevity. Mech Ageing Dev. 2013;134(9):373-380. doi:10.1016/j.mad.2013.07.001

37. Chakraborty C, Sharma AR, Sharma G, Doss CGP, Lee S-S. Therapeutic miRNA and siRNA: Moving from Bench to Clinic as Next Generation Medicine. Mol Ther Nucleic Acids. 2017;8:132-143. doi:10.1016/j.omtn.2017.06.005

\section{Supplementary Tables}

Supplemental Tables 1-3 are not available with this version.

\section{Figures}



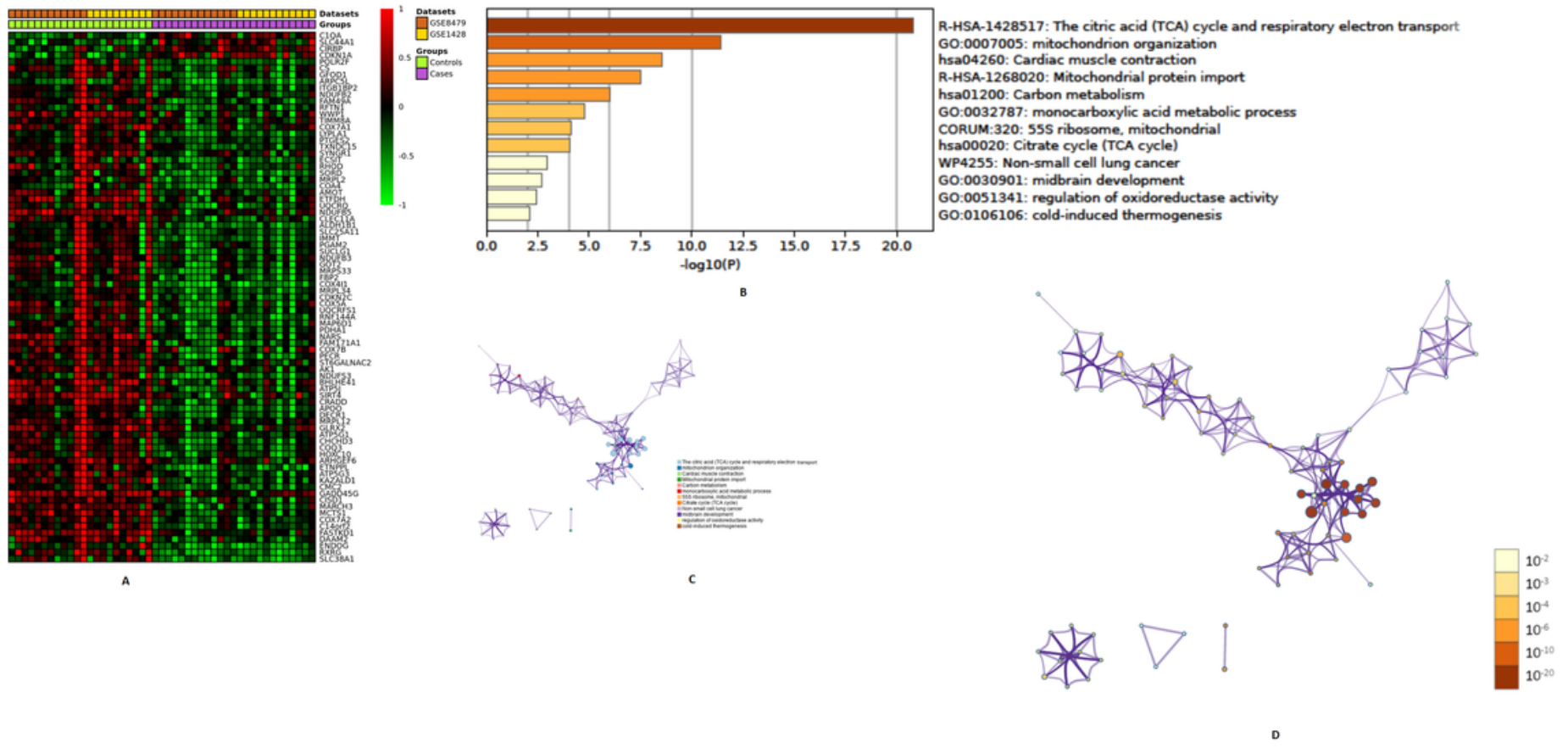

\section{Figure 1}

Identification of DEGs and pathway enrichment analysis. A) Heatmap of the top 100 genes. B) Bar graph of enriched terms across the input gene lists, colored by p-values. C) Network of enriched terms by cluster.

D) Network of enriched terms by p-value.
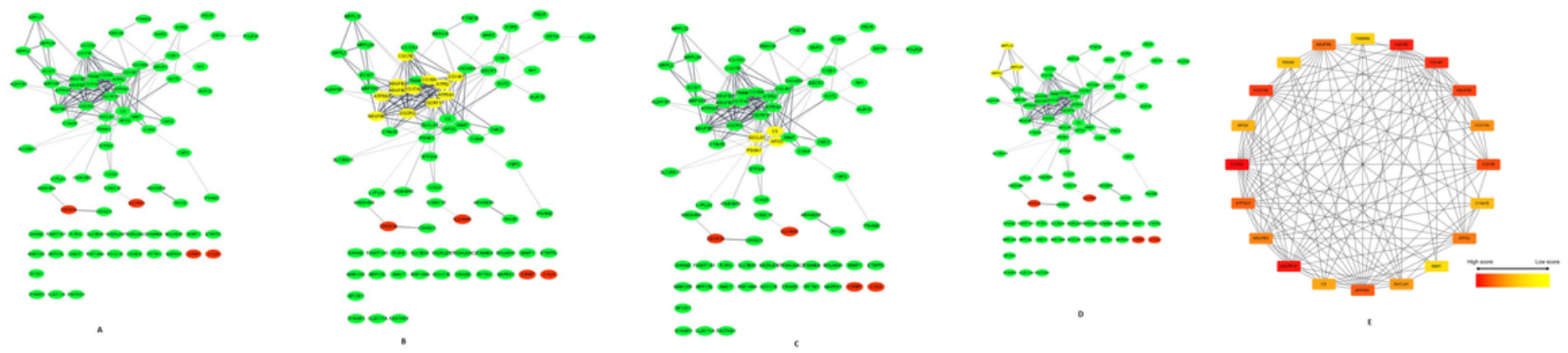

\section{Figure 2}

PPI network and module analysis. A) PPI network of DEGs constructed by STRING. Red color = upregulated genes, green color $=$ downregulated genes. B) MCODE analysis (12 nodes, 66 edges). C) MCODE analysis (4 nodes, 5 edges). D) MCODE analysis ( 3 nodes, 3 edges). E) Hub gene network of the DEGs. 

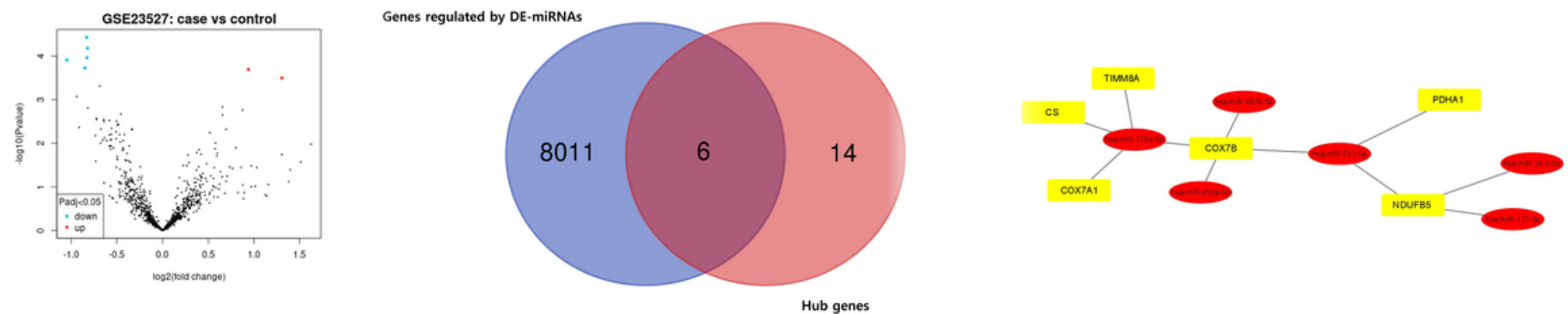

\section{Figure 3}

Prediction of potential miRNAs and target genes. A) Volcano plot of GSE23527. B) Venn diagram of regulated genes and hub genes. C) miRNA-target gene regulatory network. 